\title{
Approaches to Address NCD among PLHIV in Low and Middle Income Counties
}

\section{Ruchi Sogarwal* and Sunil Mehra}

Mamta Health Institute for Mother and Child, New Delhi-110048, India

\begin{abstract}
Human Immunodeficiency Virus (HIV) and Non Communicable Diseases (NCD) are now the major chronic diseases of public health concern, especially in low- and middle-income countries. This joint burden has major adverse effects on the quality of life of an affected individual; causes premature death; creates adverse economic effects on families in general. The purpose of this paper is to highlight extent of the problem and various approaches that can address the threat posed by NCD among people living with HIV based on evidence and best practices in health interventions. The paper is based on the non-systematic review of policies, programs, health interventions and published research articles. In addition, recommendations of expert consultations and workshops organized in various countries were also reviewed. Review indicates that there are emerging developments to bridge the gap between the NCD and HIV, however, no focused strategy is available to address NCD in PLHIV. Some of the key suggested approaches are; build evidence for planning and management of NCD in PLHIV, increase allocation of financial resources for investigation and treatment of common NCDs in PLHIV, strengthening governance structure for improved efficiency and effectiveness of systems to address NCD in HIV and non-HIV services, reconfiguration of primary health care, routine screening of PLHIV for NCD and promoting healthy lifestyle to reduce PLHIV's susceptibility to NCD, multi-/ inter-sectoral cooperation, public private partnership and community participation for sustained efforts. Although context-specific factors vary from place to place, the feasibility and cost-effectiveness of different approaches tackling NCD in PLHIV is needed.
\end{abstract}

Keywords: HIV/AIDS; Health service development; NCD; Health policy

\section{Introduction}

Human Immunodeficiency Virus (HIV) and Non Communicable Diseases $(\mathrm{NCD})$ are the major public health concern as highlighted in recent reports [1-5]. The Global Burden of Diseases study shows that low and middle income countries (LMIC) are experiencing a classic 'epidemiological transition' in which infectious diseases are still prevalent, while NCD rates (mainly cardiovascular disease, diabetes, renal diseases, chronic respiratory diseases and cancer) are rising [6]. Current research responds that PLHIV have three sources of risk of contracting NCDs, first, from HIV-related factors (including lifesaving ARV drugs) [7-9], second, pre-existing disease condition [2,9-11] or coinfection with other viruses [7,12-13], third associated with increasing age $[14,15]$. NCD in the same way as HIV affects social determinants of health directly and indirectly as it hinders ability to eradicate extreme poverty and hunger due to illness, loss of job and high cost of treatment $[3,16]$. This joint burden has major adverse effects on the quality of life of an affected individual and causes premature death. Recent evidence counters that the emerging risk of NCDs in LMICs will increase global inequalities, further exacerbated by existing social inequalities in risky behaviour in the countries facing a persistent high burden of HIV [6].

Given the similarities in key elements of prevention and management of HIV infection and NCDs (health promotion, adherence to treatment, regular monitoring of treatment outcomes, and active involvement of the client and family in care and treatment), some countries of Africa and Asia including Nigeria, Zambia, Ethiopia, Uganda, Thailand, Vietnam and Burma have piloted the integration of NCD and HIV programs on the well-established platform of HIV services under resource limited settings [17-20]. For example, in 2009, a pilot programme in Kenya [21] integrated NCD services into existing HIV comprehensive care services. In this program, HIV clients were systematically screened for NCD behavioural, biological and therapeutic risk factors. Those identified with low-to moderate risks were treated by clinicians at the comprehensive care centre, and those with moderate-to-high risks were referred to specialists. Apparent advantages have been found with the integrated models such as integration raised the awareness of NCDs among healthcare workers and improved their capacity to diagnose and treat both conditions. Furthermore, clients were contented to receive both NCD and HIV services at the same location and, in some cases, from the same health-care workers. Recently, other than integrated approach, co-location of services has also been tried out in the MSF chronic disease clinics in Cambodia which included services for HIV, diabetes and CVD and has demonstrated high acceptance and good outcomes [22]. In Lesotho, mobile clinics screen for diabetes and hypertension alongside services for HIV and antenatal care [23]. In spite of apparent advantages of these practices, most of the countries faced several challenges at system level, such as lack of organized or coordinated activities aimed at prevention and control of NCDs in PLHIV; lack of trained professionals for clinical management of NCDs in PLHIV; lack of standardized protocols or clinical guidelines; lack of system for monitoring and evaluation; and infrastructure and commodity constraints. Besides, these practices may not seem demanding to some HIV care providers in resource-constrained settings, because of the

*Corresponding author: Ruchi Sogarwal, Mamta Health Institute for Mother and Child, New Delhi, India, Tel: +91-11-29220210; Fax: +91-11-29220575; E-mail: ruchis@mamtahimc.org; ruchi.dr@gmail.com

Received April 01, 2015; Accepted June 05, 2015; Published June 15, 2015

Citation: Sogarwal R, Mehra S (2015) Approaches to Address NCD among PLHIV in Low and Middle Income Counties. J AIDS Clin Res 6: 472. doi:10.4172/21556113.1000472

Copyright: (c) 2015 Sogarwal R, et al. This is an open-access article distributed under the terms of the Creative Commons Attribution License, which permits unrestricted use, distribution, and reproduction in any medium, provided the original author and source are credited. 
urgency of identifying and getting PLHIV onto ART, and responding to the overwhelming burden of opportunistic infections and TB among PLHIV presenting late for care. Likewise, as many PLHIV avoid mainstream service systems, especially in settings where stigma and discrimination are rife or where their status or behaviours are facing taboo, special service delivery approaches are needed. Given the fact, emerging strides at various global and regional platforms [24] highlighted key questions to build the bridge between HIV and NCD, i.e. (a) What is the best and most efficient way to screen PLHIVs for NCDs in resource limited settings? (b) Where NCD prevention, care and treatment services for PLHIVs should be delivered? Should they be integrated into the HIV clinic or provided elsewhere? (c) Which health care providers should treat NCDs in PLHIV? Dialogues are on to plan a strategy to have a shared commitment and consensus on the strategic direction; to control and prevent NCDs and their common risk factors; and to provide a framework for effective and efficient coordination of activities and resource mobilization. The purpose of this paper is to highlight extent of the problem (which contains prevalence and/or incidence of common NCDs among PLHIV and associated risk factors) and various approaches that can address the threat posed by common NCDs among PLHIV based on evidence and best practices in health interventions in LMICs. The paper is based on the non-systematic review of policies, health interventions, published research articles and expert consultations. The literature and database primarily searched from MEDLINE and EMBASE, national and regional database, citation indexes using primarily Google Scholar. The literature was included if it has exclusively or predominantly assessed the burden of common NCDs on PLHIV and system response; and were published between 2000 and 2014; used rigorous designs, such as randomized controlled trials or quasi-experimental studies.

\section{Extend of the problem}

The review had focused on three common NCDs, viz., cardiovascular diseases, cancers and diabetes, which are the important comorbidities of HIV infection.

Cardiovascular Diseases (CVD): Several studies have demonstrated that PLHIV have increased risks of CVD compared to HIV-negative individuals [25-27]. This has been reported both in PLHIV on ART and in treatment naïve individuals [25-29], including children and adolescents [30]. Systematic reviews conducted in developed countries have found that the incidence of CVD in PLHIV range from 1.19 to 11.3 per 1000 person years [31]. In addition, consistent patterns of increased risk for stroke [32], sudden cardiac death [33] and heart failure [34] in PLHIV have been reported. Other studies have also demonstrated increased incidence of Coronary Heart Disease (CHD) and Myocardial Infraction in PLHIV compared to HIV-uninfected controls [35-37]. The incidence of CHD among young men (up to age 34) and women (up to age 44) with HIV infection was significantly higher than that among non-HIV-infected individuals [38]. Furthermore, the beneficial role of ART has been demonstrated in the SMART study to outweigh potential CVD risks in PLHIV [39] and in another study ART found to be cardio-protective in children and adolescents living with HIV [40]. Established CVD-risk factors have been underlined and are prevalent in PLHIV [41]; however, there is a paucity of evidence available to explain the excess risk of CVD in PLHIV in comparison to HIV uninfected populations.

Diabetes: In a systematic review reported baseline prevalence rates of $2.8 \%$ in the D:A:D study [42], $11.9 \%$ in a multi-centre AIDS Cohort study [43] and 14.9\% in the Veterans Ageing Cohort Study (VACS) [44]. In VACS study, the incidence of diabetes was significantly high and this ranged from 5.7 to 23.8 per 1000 person-years of follow-up, with role of ART implicated in most of these studies [42-44]. A multi-centre AIDS Cohort study indicates the prevalence of diabetes of $14 \%$ among those HIV positive men who were using antiretroviral treatment [43]. In a study of the prevalence of diabetes among HIV positive asymptomatic patients in Botswana, pointed out that the older age and higher BMI were associated with higher diabetes prevalence [45]. Although the association between HIV and diabetes is not fully understood, some studies have shown that the risk for diabetes is similar between PLHIV and HIV-uninfected individuals [46]. This coupled with the evidence showing that HIV-related inflammation and ARV drugs contribute to the risk of diabetes reinforces the multifactorial basis for increased prevalence of diabetes among PLHIV [46]. Other studies reported that in middle income countries such as Mexico and India, diabetes significantly increases the risk of active tuberculosis, and the comorbidity is associated with poorer outcomes [47-48].

Cancer: The incidence of non-AIDS-defining cancers has increased significantly over the past 10 years and has now surpassed that of AIDSdefining cancers in HIV-infected patients [49]. Findings from multiple studies indicated that PLHIV have a 2-3 fold increased risk of cancer compared to the general population [50]. Studies conducted in Rwanda, Kenya, South Africa, Uganda and Zambia reported prevalence of cervical pre-cancer and cancer among HIV-positive women of $24.3 \%$, $26.7 \%, 66.3 \%, 73.0 \%$ and $76 \%$, respectively [51-55]. In Botswana, the rates of non-AIDS defining events in ART-treated patients were higher than in the USA [56]. Several malignant conditions, including Hodgkin lymphoma, anal cancer, testicular cancer, lung cancer, multiple myeloma have been found in increased numbers in HIVinfected patients [57-59]. Other studies have shown that the incidence of some certain cancers are higher in adolescents living with HIV than in the general population [60-62]; and the overall mortality rate of non-AIDS defining cancers (NADCs) was higher than AIDS defining cancers (ADCs) [63]. Some authors also indicated in their review that PLHIV tend to have lung cancer at early ages even after adjusting for tobacco use in resource limited settings [64-65]. A study reported that the population-attributable fraction associated with HIV infection and smoking was approximately, 90\% [62].

In the review, most of the included studies were from the HICs and could not reflect the magnitude and determinants of NCDs of the LMICs due to paucity of data from LMIC. Though more is known about the clinical relationship between HIV and NCDs and the factors associated with their relationship, little is known about the epidemiology of their comorbidity. With the emergence of double mortality burden in LMIC, better understanding of the epidemiology of HIV-NCD comorbidity is essential [66].

\section{Approaches to address NCD among PLHIV}

Overall, there are three types of PLHIV, viz., those who are on first line ART, those who are on second line ART; those who are yet not eligible for ART. Hence, to strengthen NCD care in PLHIV, the specific approaches suggested for LMIC are described below, which are based on the evidence and best practices in heath interventions:

Build evidence for proper planning of prevention and control strategies of NCD among PLHIV: Proper planning and management of prevention and control strategies depend on the availability of reliable and comparable information. The step wise approach that explores the most practical and efficient methods of applying existing knowledge is crucial for interventions essential for achieving policies for chronic diseases. Hence, the approach should focus on building the evidence 
on NCD in PLHIV in LMIC. Some of the research areas [67] that may require focus are (a) defining the burden of NCDs among PLHIV, (b) assessment of health system to monitor the gap in providing NCD care and treatment to PLHIV, (c) evaluate effective and efficient strategies at individual and health system levels. On priority, optimal use of existing data sources should be made, for example by linking routine HIV clinical databases to laboratory databases, cancer registries, medical and hospitalization records etc. In addition, for NCDs with sufficient high prevalence and incidence, sub-studies can be added to the large prospective studies that are addressing other questions.

Proper allocation of financial resources for investigation and treatment of common NCDs in PLHIV: HIV/AIDS received global attention following high level meeting in the UN General Assembly in the year 2000 [68]. This led to a global movement to mobilize financial resources to address three key communicable diseases; i.e. Global Funds for AIDS, TB and Malaria. This was one major step to convert policy into action. Likewise, due to emerging trend and its serious impact on health, NCD also received global attention succeeding the second meeting of UN General Assembly (2011) [69]. Many LMICs have developed their action plans for NCD by Government without or with external financial contributions; however, there is no recommendation or policy guideline on mobilizing funds for addressing NCDs and their risk factors in PLHIV. There has been indifferent response from multilateral bodies like WHO, World Bank or from International organizations for funding NCDs. Hence, it is worthwhile to explore different ways to mobilize financial resources for NCD care among PLHIV through either additional budgeting within HIV program involving external contributors or common pool funds from both NCD and HIV programs or mobilizing fund through service user charges and treatment on subsidized rates from PLHIV who can afford.

\section{Strengthening governance structure for improved efficiency and effectiveness of systems to address NCD in HIV and non- HIV services}

Though there has been a dramatic rise in expenditure on HIV and NCD programmes in the last five years but this is accompanied by growing challenges about implementation. An important reason for the relative lack of success of many initiatives in addressing the problem in LMIC is that the local institutions are not adequately empowered. Without reforms in governance structures, policy and programs aimed to address NCD in people with or without HIV infection will continue to be afflicted. Hence, a broad range of reforms needed in the areas enumerated such as strengthening local Institutions; building quality in service delivery; partnership with civil society including NGOs, professional institutions and other community based institutions; coordination and streamlining of approval processes in delivering holistic outcomes.

\section{Reconfiguring primary care and strengthening management of NCD in PLHIV}

LMIC need to have strong and dynamic health systems that can respond effectively to changes in the epidemiologic pattern of diseases. These countries cannot afford to address diseases turn by turn. Therefore, the future direction of health care system should be on integrated management rather than management of a single disease. At the point of service delivery, following models are suggested:

o Model-1 (e.g. Ethiopia) could be a shared clinic that has support staff in common, but which dedicates different days to different chronic diseases, for example seeing HIV patients on Mondays and diabetic patients on Tuesdays. In the model, the systems (e.g. charting tools, appointment systems, adherence support and $M \leftrightarrow E$ protocols) could be identical, but the clinical care would be disease-specific $[70,44]$.

o Model-2 (e.g. Cambodia model) could be to cross-trained the health workforce (physicians and other support staff including nurses, pharmacist, lab technicians, counselors) to investigate and manage HIV and NCDs at the same time [22].

o Model-3 (e.g. India model), PHIV with NCD could be seen at parallel clinics - or in HIV clinic on the one hand, and OPD on the other. In India both HARRT and NCDs are generally managed by Physicians in the Department of Internal Medicine [71-72].

o Other way, could be providing converting HAART clinics into 'Lifestyle clinics' or 'Chronic Disease Clinics', in this model a comprehensive services would be provided to PHIV under one umbrella.

There is no right way to address this; instead it's about what works best in particular setting. Routine screening of PLHIV for NCD and promote healthy
lifestyle to reduce PLHIV's susceptibility to NCD

As HIV treatment scale-up continues, an increasing number of PLHIV will live long enough to develop NCDs and other chronic diseases. Currently millions of PLHIV are enrolled in care and treatment programs in LMIC and more than 5 million of them are on ART [73]. Furthermore, metabolic complications of HIV and some ARVs increase the risk of other chronic diseases and risk factors. Thus, it is vital to routinely screen PLHIV for NCD and their risk factors (e.g. blood pressure, blood sugar levels and cervical cancer) and promote healthy lifestyle behavior. This would help to reduce PLHIV's susceptibility to NCD.

\section{Emphasis on community participation for improved access to information and services for NCD care in PLHIV}

It is well proven that the effectiveness of healthcare system is affected by the ability of the community itself to participate in service delivery. The opportunity to design and manage such delivery provides empowerment to the community as well as better access, accountability and transparency [74]. Hence, following key dimensional ways are suggested to increase community participation for improved access to information and services for NCD care in PLHIV:

Empower local NGOs, Self-help Groups and community based institutions with knowledge on risky behaviour, signs and symptoms, risk factor detection, availability of services, home based care etc.

$\bigcirc$ Increase patient's and their families' participation in preparing personalised action plans to minimize the risk of NCDs, while addressing the issue of social development.

Ensure PLHIV rights to NCD care and treatment services.

Multi-/inter-sectoral cooperation and public private partnership for effective and sustain efforts

Multi-/inter-sectoral actions are necessary at all stages of policy formulation and implementation because major determinants of HIV and NCDs lie outside the health sector (e.g. education, labour). 
Given the scale of the NCDs with/without HIV infection, public and private partnership(PPP) is essential to sustain efforts of the magnitude required to respond to the NCD challenge in LMIC. Hence, following areas are suggested for comprehensive public-health action where multi-/inter-sectoral cooperation and PPP can help to effectively address NCD care in PLHIV:

Universal access to services for people at risk and those with HIV and/or NCD

O Adherence to medication

Uninterrupted medical supply chain

Family focused care and self-management

Linkages and referrals within / between the health facility, and between facility and community

\section{Conclusion}

The common NCDs irrespective of the HIV status are priority now in most LMIC, and it would be a serious gaffe to ignore it in the coming decade. The body of evidence relating to country experiences with both positive and negative outcomes will be essential to providing clearer guidance and indicators on how best to address NCD in PHIV within wider health and development efforts. In recognition that most LMIC will not have the resources to immediately provide comprehensive solutions, approaches that are feasible and likely to have the greatest impact for the investment are selected first for implementation. Although context-specific factors vary from place to place, the feasibility and cost-effectiveness of different management and service delivery models is needed before considering adoption of single and/or multiple approaches in LMIC. Hence, it would be useful for international agencies, governments and other stakeholders to debate and produce a common agenda for action on NCDs in PLHIV focused on their causes, prevention, and control within the context of the health care system. Besides, there is need for systematic reviews of available evidences and new research to addressing the knowledge gap in LMIC.

\section{Reference}

1. Leeder S, Raymond S, Greenberg H, Liu H, Esson K (2004) A Race Against time: The challenge of cardiovascular disease in developing economies. The Earth Institute, Columbia University; New York.

2. World Health Organization (2005) Preventing chronic diseases: A vital investment. WHO, Geneva.

3. Suhrcke M, Nugent RA, Stuckler D, Rocco L (2006) Chronic disease: an economic perspective. Oxford Health Alliance, London.

4. Gaziano TA, Galea G, Reddy KS (2007) Scaling up interventions for chronic disease prevention: the evidence. Lancet 370: 1939-1946.

5. Ollila E (2005) Global health priorities - priorities of the wealthy? Global Health 1: 6 .

6. GBD (2013) Global, regional, and national age-sex specific all-cause and cause-specific mortality for 240 causes of death, 1990-2013: a systematic analysis for the Global Burden of Disease Study 2013. The Lancet 385: 117171.

7. Kalra S, Agrawal N (2013) Diabetes and HIV: current understanding and future perspectives. Curr Diab Rep 13: 419-427.

8. Kiage JN, Heimburger DC, Nyirenda CK, Wellons MF, Bagchi S, et al. (2013) Cardiometabolic risk factors among HIV patients on antiretroviral therapy. Lipids Health Dis 12: 50.

9. Triant VA (2013) Cardiovascular disease and HIV infection. Curr HIVIAIDS Rep 10: 199-206.

10. Hemkens LG, Bucher HC (2014) HIV infection and cardiovascular disease. Eur Heart J 35: 1373-1381.
11. Winston J, Deray G, Hawkins T, Szczech L, Wyatt C, et al. (2008) Kidney disease in patients with HIV infection and AIDS. Clin Infect Dis 47: 1449-1457.

12. Berg CJ, Michelson SE, Safren SA (2007) Behavioral aspects of HIV care: adherence, depression, substance use, and HIV-transmission behaviors. Infect Dis Clin North Am 21: 181-200, x.

13. Helleberg M, Gerstoft J, Afzal S, Kronborg G, Larsen CS, et al. (2014) Risk of cancer among HIV-infected individuals compared to the background population: impact of smoking and HIV. AIDS 28: 1499-1508.

14. Kagaruki GB, Mayige MT, Ngadaya ES, Kimaro GD, Kalinga AK, et al. (2014) Magnitude and risk factors of non-communicable diseases among people living with HIV in Tanzania: a cross sectional study from Mbeya and Dar es Salaam regions. BMC Public Health 14: 904.

15. http://www.aidsmap.com/HIV-and-non-communicable-diseases-NCDs/ page/2094965/.

16. http://www.aidsmap.com/HIV-and-non-communicable-diseases-NCDs/ page/2094965/\#item2094967.

17. Braitstein P, Einterz RM, Sidle JE, Kimaiyo S, Tierney W (2009) "Talkin' about a revolution": How electronic health records can facilitate the scale-up of HIV care and treatment and catalyze primary care in resource-constrained settings. J Acquir Immune Defic Syndr 52 Suppl 1: S54-57.

18. Global Health Council (2011) HIV and NCDs: leveraging HIV scale up to strengthen chronic disease services in LMIC. Meeting session, 38th Annual International Conference on Global Health, Washington, DC.

19. Global Health Council (2011) Tackling NCDS: how can existing platforms be leveraged? Panel Session, New York, 18 September.

20. Lamptey $P$, Dirks $R$ (2012) Building on the AIDS response to tackle noncommunicable disease. Glob Heart 7: 67-71.

21. http://www.unaids.org/en/media/unaids/contentassets/documents/ unaidspublication/2011/20110526_JC2145_Chronic_care_of_HIV.pdf .

22. Janssens B, Van Damme W, Raleigh B, Gupta J, Khem S, et al. (2007) Offering integrated care for HIVIAIDS, diabetes and hypertension within chronic disease clinics in Cambodia. Bull World Health Organ 85: 880-885.

23. Tiam A, Oyebanji O, Nkonyana J, Ahimbisibwe A, Putsoane M, et al. (2012) Family Health Days: An innovative approach to providing integrated health services for HIV and non-communicable diseases among adults and children in hard-to-reach areas of Lesotho. In: International AIDS Conference. Washington, USA.

24. http://pag.aids2012.org/session.aspx?s=230.

25. Triant VA (2013) Cardiovascular disease and HIV infection. Curr HIVIAIDS Rep 10: 199-206.

26. Hemkens LG, Bucher HC (2014) HIV infection and cardiovascular disease. Eur Heart J 35: 1373-1381.

27. Islam FM, Wu J, Jansson J, Wilson DP (2012) Relative risk of cardiovascular disease among people living with HIV: a systematic review and meta-analysis. HIV Med 13: 453-468.

28. Garg H, Joshi A, Mukherjee D (2013) Cardiovascular complications of HIV infection and treatment. Cardiovasc Hematol Agents Med Chem 11: 58-66.

29. Thienemann F, Sliwa K, Rockstroh JK (2013) HIV and the heart: the impact of antiretroviral therapy: a global perspective. Eur Heart J 34: 3538-3546.

30. http://circ.ahajournals.org/content/early/2013/12/23/CIRCULATIONAHA.113.001978.

31. Nigatu T, Brian O, Julian E, Geoffrey S, Mulu AW (2013) The incidence of cardiovascular disease, cancer and type 2 diabetes comorbidities in HIV infection: A systematic review. Journal of Nursing Education and Practice, 3: 58-67.

32. Chow FC, Regan S, Feske S, Meigs JB, Grinspoon SK, et al. (2012) Comparison of ischemic stroke incidence in HIV-infected and non-HIV-infected patients in a US health care system. J Acquir Immune Defic Syndr 60: 351-358.

33. Tseng ZH, Secemsky EA, Dowdy D, Vittinghoff E, Moyers B, et al. (2012) Sudden cardiac death in patients with human immunodeficiency virus infection. J Am Coll Cardiol 59: 1891-1896.

34. Butt AA, Chang CC, Kuller L, Goetz MB, Leaf D, et al. (2011) Risk of heart failure with human immunodeficiency virus in the absence of prior diagnosis of 
Citation: Sogarwal R, Mehra S (2015) Approaches to Address NCD among PLHIV in Low and Middle Income Counties. J AIDS Clin Res 6: 472. doi:10.4172/2155-6113.1000472

Page 5 of 5

coronary heart disease. Arch Intern Med 171: 737-743.

35. Currier JS, Taylor A, Boyd F, Dezii CM, Kawabata H, et al. (2003) Coronary heart disease in HIV-infected individuals. J Acquir Immune Defic Syndr 33: 506-512.

36. Obel N, Thomsen HF, Kronborg G, Larsen CS, Hildebrandt PR, et al. (2007) Ischemic heart disease in HIV-infected and HIV-uninfected individuals: a population-based cohort study. Clin Infect Dis 44: 1625-1631.

37. Triant VA, Lee H, Hadigan C, Grinspoon SK (2007) Increased acute myocardial infarction rates and cardiovascular risk factors among patients with human immunodeficiency virus disease. J Clin Endocrinol Metab 92.

38. Currier JS, Taylor A, Boyd F, Dezii CM, Kawabata H, et al. (2003) Coronary heart disease in HIV-infected individuals. J Acquir Immune Defic Syndr 33: 506-512.

39. Strategies for Management of Antiretroviral Therapy (SMART) Study Group1, El-Sadr WM, Lundgren J, Neaton JD, Gordin F, et al. (2006) CD4+ countguided interruption of antiretroviral treatment. N Engl J Med 355: 2283-2296.

40. Lipshultz SE, Williams PL, Wilkinson JD, Erin CL, Russell BV, et al. (2013) Cardiac status of children infected with human immunodeficiency virus who are receiving long-term combination antiretroviral therapy: results from the Adolescent Master Protocol of the Multicenter Pediatric HIVIAIDS Cohort Study. JAMA Pediatrics 167: 520-7.

41. So-Armah K, Freiberg MS (2014) Cardiovascular disease risk in an aging HIV population: not just a question of biology. Curr Opin HIV AIDS 9: 346-354.

42. De Wit S, Sabin CA, Rainer W, Signe WW, Peter R, et al. (2008) Incidence and risk factors for new-onset diabetes in HIV-infected patients: the data collection on adverse effects of anti-HIV drugs (D:A:D) study. Diabetes Care 31: 12241229.

43. Brown TT, Cole SR, Li X, Kingsley LA, Palella FJ, et al. (2005) Antiretroviral therapy and the prevalence and incidence of diabetes mellitus in the multicenter AIDS cohort study. Arch Intern Med 165: 1179-1184.

44. Haregu TN, Oldenburg B, Setswe G, Elliott J (2012) Magnitude of Diabetes comorbidity among people living with HIV: Systematic Review. International Journal of Diabetes Research 1: 81-86.

45. Irene H, Marianna KB, Fatma H, Hermann B, Priti D, Joe M et al. (2009) Diabetes in HIV Positive Adults in Botswana: Nutritional and Demographic Characteristics. FASEB J 23 (Meeting Abstract Supplement) 547.15.

46. Kalra S, Kalra B, Agrawal N, Unnikrishnan A (2011) Understanding diabetes in patients with HIVIAIDS. Diabetol Metab Syndr 3: 2.

47. Young F, Critchley JA, Johnstone LK, Unwin NC (2009) A review of comorbidity between infectious and chronic disease in Sub Saharan Africa: TB and diabetes mellitus, HIV and metabolic syndrome, and the impact of globalization. Global Health 5: 9.

48. Harries AD, Lin Y, Satyanarayana S, Lönnroth K, Li L, et al. (2011) The looming epidemic of diabetes-associated tuberculosis: learning lessons from HIV-associated tuberculosis. Int J Tuberc Lung Dis 15: 1436-1444, i.

49. Mitsuyasu RT (2008) Non--AIDS-defining malignancies in HIV. Top HIV Med 16: 117-121.

50. Ruiz M (2009) Certain non-AIDS-defining cancers higher in HIV population. HIV Clin 21: 13-16.

51. Leroy V, Ladner J, De Clercq A, Meheus A, Nyiraziraje M, et al. (1999) Cervica dysplasia and HIV type 1 infection in African pregnant women: a cross sectional study, Kigali, Rwanda. The Pregnancy and HIV Study Group (EGE). Sex Transm Infect 75: 103-106.

52. Memiah P, Mbuthia W, Kiiru G, Agbor S, Odhiambo F, et al. (2012) Prevalence and Risk Factors Associated with Precancerous Cervical Cancer Lesions among HIV-Infected Women in Resource-Limited Settings. AIDS Res Treat 2012: 953743.

53. Moodley J, Constant D, Hoffman M, Salimo A, Allan B, et al. (2009) Human papillomavirus prevalence, viral load and pre-cancerous lesions of the cervix in women initiating highly active antiretroviral therapy in South Africa: a crosssectional study. BMC Cancer 9: 275.

54. Blossom DB, Beigi RH, Farrell JJ, Mackay W, Qadadri B, et al. (2007) Human papillomavirus genotypes associated with cervical cytologic abnormalities and HIV infection in Ugandan women. J Med Virol 79: 758-765.
55. Parham GP, Sahasrabuddhe VV, Mwanahamuntu MH, Shepherd BE, Hicks $\mathrm{ML}$, et al. (2006) Prevalence and predictors of squamous intraepithelial lesions of the cervix in HIV-infected women in Lusaka, Zambia. Gynecol Oncol 103 1017-1022.

56. Wester CW, Jhon Rk, Bryan ES, Samuel ES, Peter FR, et al. (2011) NonAIDS defining events among HIV-1 infected adults receiving combination antiretroviral therapy In urban settings in Sub-Saharan Africa and the United States. AIDS 25: 1471-1479.

57. Long JL, Engels EA, Moore RD, Gebo KA (2008) Incidence and outcomes of malignancy in the HAART era in an urban cohort of HIV-infected individuals. AIDS 22: 489-496.

58. Burgi A, Stephanie B, Scott W, Mark M, Mark RW, Katherine S, et al. (2005) Incidence and risk factors for the occurrence of non-AIDS-defining cancer among human immunodeficiency virus-infected individuals. Cancer 104: Number 7.

59. Chiao EY, Krown SE (2003) Update on non-acquired immunodeficiency syndrome-defining malignancies. Curr Opin Oncol 15: 389-397.

60. Davidson A, Wainwright RD, Stones DK, Kruger M, Hendricks M, et al. (2014) Malignancies in South African children with HIV. J Pediatr Hematol Oncol 36: 111-117.

61. Simard EP, Shiels MS, Bhatia K, Engels EA (2012) Long-term cancer risk among people diagnosed with AIDS during childhood. Cancer Epidemiol Biomarkers Prev 21: 148-154.

62. Helleberg M, Afzal S, Kronborg G, Larsen CS, Pedersen G, et al. (2013) Mortality attributable to smoking among HIV-1-infected individuals: a nationwide, population-based cohort study. Clin Infect Dis 56: 727-734.

63. Monforte Ad, Abrams D, Pradier C, Weber R, Reiss P, et al. (2008) HIVinduced immunodeficiency and mortality from AIDS-defining and non-AIDSdefining malignancies. AIDS 22: 2143-2153.

64. Hirschhorn LR, Kaaya SF, Garrity PS, Chopyak E, Fawzi MC (2012) Cance and the 'other' noncommunicable chronic diseases in older people living with HIVIAIDS in resource-limited settings: a challenge to success. AIDS 26 Suppl 1: S65-75.

65. Reynolds NR, Neidig JL, Wewers ME (2004) Illness representation and smoking behavior: a focus group study of HIV-positive men. J Assoc Nurses AIDS Care 15: 37-47.

66. Misganaw A, Mariam DH, Araya T (2012) The double mortality burden among adults in Addis Ababa, Ethiopia, 2006-2009. Prev Chronic Dis 9: E84.

67. Petersen M, Yiannoutsos CT, Justice A, Egger M (2014) Observational research on NCDs in HIV-positive populations: conceptual and methodological considerations. J Acquir Immune Defic Syndr 67 Suppl 1: S8-16.

68. http://www.un.org/millennium/declaration/ares552e.pdf.

69. http://www.un.org/en/ga/ncdmeeting2011/.

70. Rabkin M, Kruk ME, El-Sadr WM (2012) HIV, aging and continuity care: strengthening health systems to support services for noncommunicable diseases in low-income countries. AIDS 26 Suppl 1: S77-83.

71. http://www.gujhealth.gov.in/images/npcdcs.pdf

72. http://www.naco.gov.in/upload/Publication/Treatment $\% 20$ Care $\% 20$ and $\% 20$ support/Operational\%20guidelines\%20for\%20ART\%20services.pdf.

73. http://www.aids2014.org/webcontent/file/AIDS2014_Global_Factsheet_ April_2014.pdf.

74. http://www.unaids.org/sites/default/files/media_asset/20120628_JC2281_ PromisingPracticesCommunityEngagements en 0.pdf. 